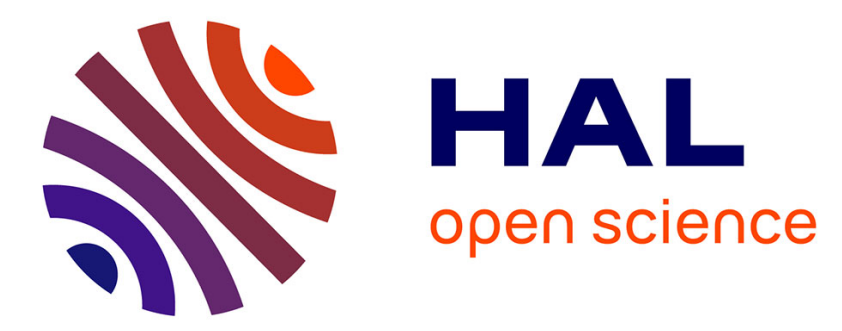

\title{
Multiscale Stick-Slip Dynamics of Adhesive Tape Peeling
} Marie-Julie Dalbe, Pierre-Philippe Cortet, Matteo Ciccotti, Loïc Vanel, Stéphane Santucci

\section{To cite this version:}

Marie-Julie Dalbe, Pierre-Philippe Cortet, Matteo Ciccotti, Loïc Vanel, Stéphane Santucci. Multiscale Stick-Slip Dynamics of Adhesive Tape Peeling. Physical Review Letters, 2015, 115, pp.128301. 10.1103/PhysRevLett.115.128301 . hal-01370477

\section{HAL Id: hal-01370477 https://hal.science/hal-01370477}

Submitted on 22 Sep 2016

HAL is a multi-disciplinary open access archive for the deposit and dissemination of scientific research documents, whether they are published or not. The documents may come from teaching and research institutions in France or abroad, or from public or private research centers.
L'archive ouverte pluridisciplinaire HAL, est destinée au dépôt et à la diffusion de documents scientifiques de niveau recherche, publiés ou non, émanant des établissements d'enseignement et de recherche français ou étrangers, des laboratoires publics ou privés. 


\title{
Multiscale Stick-Slip Dynamics of Adhesive Tape Peeling
}

\author{
Marie-Julie Dalbe, ${ }^{1,2}$ Pierre-Philippe Cortet, ${ }^{3}$ Matteo Ciccotti,${ }^{4}$ Loïc Vanel,${ }^{2}$ and Stéphane Santucci ${ }^{1, *}$ \\ ${ }^{1}$ Laboratoire de Physique de l'École Normale Supérieure de Lyon, CNRS and Université de Lyon, 69364 Lyon, France \\ ${ }^{2}$ Institut Lumière Matière, UMR5306 Université Lyon 1-CNRS, Université de Lyon, 69622 Villeurbanne, France \\ ${ }^{3}$ Laboratoire FAST, CNRS, Université Paris-Sud,91405 Orsay, France \\ ${ }^{4}$ Laboratoire SIMM, UMR7615 ESPCI-CNRS-UPMC-PSL, 75231 Paris, France
}

(Received 9 April 2015; revised manuscript received 30 July 2015; published 15 September 2015)

\begin{abstract}
Using a high-speed camera, we follow the propagation of the detachment front during the peeling of an adhesive tape from a flat surface. In a given range of peeling velocity, this front displays a multiscale unstable dynamics, entangling two well-separated spatiotemporal scales, which correspond to microscopic and macroscopic dynamical stick-slip instabilities. While the periodic release of the stretch energy of the whole peeled ribbon drives the classical macro-stick-slip, we show that the micro-stick-slip, due to the regular propagation of transverse dynamic fractures discovered by Thoroddsen et al. [Phys. Rev. E 82, 046107 (2010)], is related to a high-frequency periodic release of the elastic bending energy of the adhesive ribbon concentrated in the vicinity of the peeling front.

DOI: 10.1103/PhysRevLett.115.128301

PACS numbers: 82.35.Gh, 62.20.mm, 68.35.Np
\end{abstract}

Understanding the velocity at which fractures propagate is a key issue in various fields, ranging from mechanical engineering to seismology. When fracture energy becomes a decreasing function of crack velocity (i.e., it costs less energy for a crack to grow faster), a dynamical instability can occur, leading to crack velocity periodic oscillations, as, for example, in friction problems [1]. During these oscillations, transitions from quasistatic to dynamic crack propagation regimes may then develop [2], the latter being prone to trigger fracture front instabilities [3]. The stick-slip oscillations of the detachment front during the peeling of adhesive tapes is another outstanding example, extensively studied [4-15]. However, it remains nowadays an industrial concern, leading to unacceptable noise levels, damage to the adhesive, and mechanical problems on assembly lines. Thanks to technological progress in high-speed imaging, direct observations of the peeling front dynamics in the stick-slip regime revealed a complex dynamical and multiscale process [16-20], challenging our current understanding. In particular, Thoroddsen et al. [16] observed a regular substructure of a few hundred microns wide transverse bands, formed during the "slip" phase of the stick-slip oscillations by a periodic propagation of rapid fractures across the tape width, intrinsically akin to a dynamic fracture instability [3].

In this Letter, we unveil the physical mechanism of this instability. We show that the micro-stick-slip dynamics of the detachment front, due to the high-frequency periodic propagation of transverse fractures, can be observed (i) for imposed peeling velocities in the macroscopic stick-slip domain, during the slip phase of the macroscopic instability, but also (ii) for imposed peeling velocities in a finite range beyond the macroscopic stick-slip domain, where the peeling is rapid and regular at the macroscopic scale. In the former case, we confirm here the entanglement of the microscopic and macroscopic dynamical instabilities leading to a complex multiscale stick-slip dynamics $[16,18]$. In contrast with the macroscopic stick-slip, we find that the high-frequency micro-stick-slip has an amplitude and a period independent of the peeled tape length $L$ between the detachment front and the point at which the traction is applied. Thanks to time-resolved measurements of the peeled tape Elastica profiles, we show that the microscopic instability is driven by a periodic release of the ribbon bending energy concentrated in the vicinity of the peeling front. From the measurements of the bending energy released during a microslip, we could estimate the effective peeling fracture energy in the micro-stick-slip regime, in agreement with direct macroscopic measurements [11].

We peel an adhesive tape from a microscope plate by winding its extremity at a constant velocity $V$ using a brushless motor. The winding cylinder is at a distance $L$ from the substrate and the peeling angle is set to $\theta \simeq 90^{\circ}$ (Fig. 1). A high-speed camera (Photron SA5) mounted on a microscope images the substrate-adhesive interface over about $1 \mathrm{~mm}^{2}$ at a fixed spatial resolution of $9.5 \mu \mathrm{m} /$ pixel. Depending on the imposed peeling velocity $V$, the frame rate is varied between 300000 frames/s for $256 \times 64$ pixel $^{2}$ images and 525000 frames/s for $192 \times 32$ pixel $^{2}$ images. During an experiment, the peeling angle $\theta$ and length $L$ vary less than $4 \%$ and $0.2 \%$, respectively, and are thus considered constant. We use 3M Scotch ${ }^{\circledR} 600$ tape $[11,14,17,19,20]$ made of a polyolefin blend backing $(e=34 \mu \mathrm{m}$ thick, $b=19 \mathrm{~mm}$ wide, tensile modulus $E=1.41 \pm 0.11 \mathrm{GPa}$ ) coated with a $20 \mu \mathrm{m}$ layer of a synthetic acrylic adhesive, peeled from a plate previously covered by one layer of Scotch 600 tape with its backing release coating cleansed with ethanol.

Analyzing the grey levels of the recorded images (Fig. 1, left), we extract the longitudinal position of the detachment 


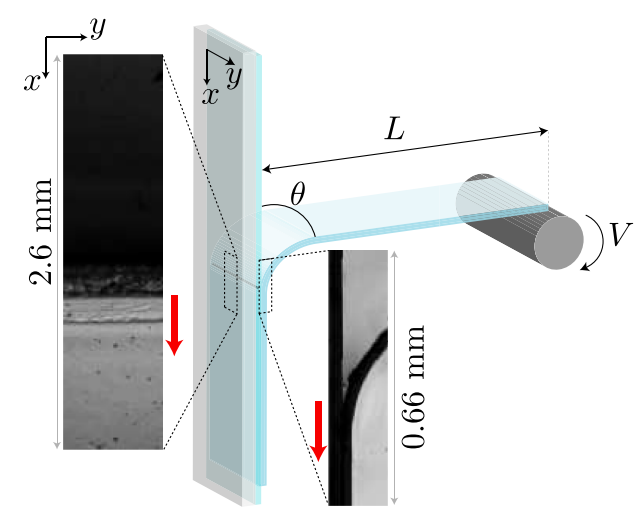

FIG. 1 (color online). Microscopic observations of the detachment front and ribbon profile when peeling a tape from a flat surface at a velocity $V$, a distance $L$, and an angle $\theta \simeq 90^{\circ}$. The arrows show the propagation direction.

front $x\left(y_{0}, t\right)$ at a given transverse position $y_{0}$. Figure 2 gives a typical example of such a local front position time series [21]. While the average front velocity over the experiment duration is equal to the driving velocity $V$ (dashed line in Fig. 2), we observe that the peeling front advances by steps, characteristics of the stick-slip instability. We could observe this standard macroscopic instability with periods of the front velocity oscillations of a few to a few tens of milliseconds over a finite range of driving velocity $V \in\left[V_{a}, V_{d}\right][20]$. In this range, the limit cycles of the macroscopic instability progressively change from typical stick-slip relaxations to nearly sinusoidal oscillations, controlled by the peeled tape inertia [20]. The instability onset observed here at $V_{a}=0.35 \pm$ $0.05 \mathrm{~m} \mathrm{~s}^{-1}$ corresponds to the velocity at which the fracture energy $\Gamma$ of the adhesive-substrate joint starts to decrease with $V$ [22]. For $V>V_{d}=5.6 \pm 1.4 \mathrm{~m} \mathrm{~s}^{-1}$, we observe anew a stable peeling at the millisecond time scale [20]. During the rapid slip phases, we observe a secondary

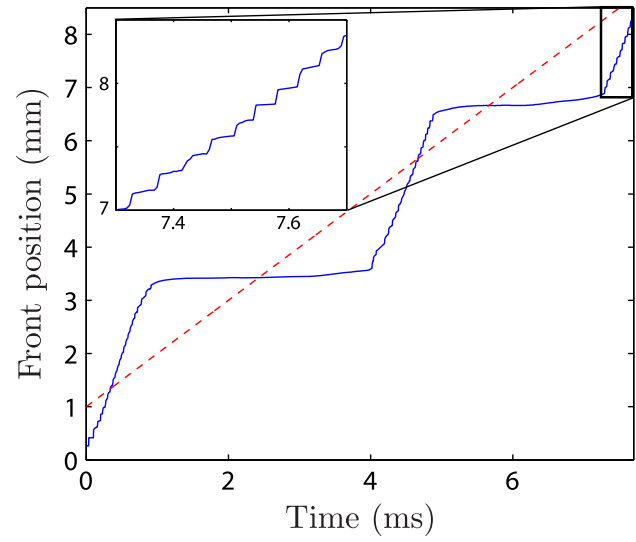

FIG. 2 (color online). Local peeling front position (in direction $x$ ) as a function of time for $L=80 \mathrm{~cm}$ and $V=1 \mathrm{~m} \mathrm{~s}^{-1}$, showing two macro-stick-slips. A zoom on a slip event reveals the microstick-slip instability. The slope of the dashed line is the driving velocity $V$. regular steplike dynamics at much shorter spatial and temporal scales, as shown in Fig. 2. Pushing the acquisition rate up to 700000 frames/s, we observe [21] that this microscopic stick-slip dynamics of the local peeling front position is due to the periodic propagation of fractures at the substrate-adhesive interface in the direction $y$ transverse to the peeling direction $x$. The width (in direction $x$ ) of the adhesive bands detached from the substrate by the propagation of those transverse fractures sets the micro-stick-slip amplitude $A_{m s s}$. Such regular propagation of rapid fractures during the slip phase of the peeling instability was discovered by Thoroddsen et al. [16]. In contrast to our experiments, they report tilted peeling fronts (up to $30^{\circ}$ ) with respect to the transverse direction $y$, probably due to their manual pulling procedure. We estimate similar transverse fracture velocities from $650 \mathrm{~m} \mathrm{~s}^{-1}$ up to $900 \mathrm{~m} \mathrm{~s}^{-1}$ confirming the dynamic nature of this rupture process.

Contrary to the macroinstability, the driving velocity $V$ is not a relevant parameter to determine the presence of the microinstability, since for $V \in\left[V_{a}, V_{d}\right]$ the micro-stick-slip is observed during the macroslips but not during the macrosticks. A possible order parameter for this microinstability is the peeled tape velocity beyond the tape bended section close to the detachment front. We estimate this velocity by measuring the detachment front velocity $v_{m}(t)=\int_{t}^{t+\tau} \dot{x}\left(y_{0}, t^{\prime}\right) d t^{\prime} / \tau$ averaged at a time scale $\tau$ of a few hundreds of $\mu \mathrm{s}$, intermediate between the time scales of the macro- and micro-stick-slip dynamics. The separation of those time scales by at least 1.5 order of magnitude in our experiments ensures the robustness of the observable $v_{m}(t)$. For $V \notin\left[V_{a}, V_{d}\right]$, the peeling is macroscopically stable at the ms time scale. At the $\mu$ s time scale, a stable peeling is still observed, with $\dot{x}(t) \simeq v_{m}(t)=V$, when $V<V_{a}$ or $V>v_{d}=21.1 \pm 3.1 \mathrm{~m} \mathrm{~s}^{-1}$. However, for $V \in\left[V_{d}, v_{d}\right]$, the micro-stick-slip is observed, such that $\dot{x}(t) \neq v_{m}(t)$ and $v_{m}(t)=V$. For $V \in\left[V_{a}, V_{d}\right]$, the front alternatively propagates either at a velocity $v_{m}(t)$ smaller than $V_{a}$ (during the macrostick phase) for which peeling is stable at the $\mu$ s scale $\left[\dot{x}(t) \simeq v_{m}(t)\right]$, or with a high-frequency jerky dynamics - the micro-stick-slip-of mean velocity $v_{m}(t)$ larger than $V_{a}$ (during the macroslip phase, see the inset of Fig. 2). As a result, when $V_{a}<v_{m}(t)<v_{d}$, the front always displays micro-stick-slips, independently of the macroscopic peeling regime (see the state diagram in the Supplemental Material [21]). Thus, we report that the macro- and microinstabilities appear for similar velocities, while they disappear at significantly different ones, $V_{d}$ and $v_{d}\left(>V_{d}\right)$, respectively. Interestingly, the characteristic velocity $v_{d}$ at which the micro-stick-slip disappears is close to the velocity $V_{0} \sim 19 \mathrm{~m} \mathrm{~s}^{-1}$ [11] above which the fracture energy of the adhesive-substrate joint $\Gamma$ is increasing anew with the front velocity $v_{m}$. Our results tend to show that, while the macro-stick-slip instability does not develop over the whole decreasing range of $\Gamma[20,23]$, the micro-stick-slip instability seems to occur as soon as the 
velocity $v_{m}(t)$ of the detachment front belongs to this decreasing branch of $\Gamma$, and thus, is fundamentally a dynamic fracture instability.

For a set of parameters $(L, V)$, we extract amplitude and duration of at least a hundred of micro-stick-slip cycles from a dozen of experiments. We compute the mean amplitude $A_{\mathrm{mss}}$, mean duration $T_{\mathrm{mss}}$, and corresponding mean front velocity $v_{m}$. Contrary to the macroinstability, where durations and amplitudes of the cycles are proportional to $L[8,19]$, the micro-stick-slips features appear not to depend significantly on $L$ (Fig. 4). The instability amplitude $A_{m s s}$ is, moreover, nearly constant with $v_{m}$ with a mean value $\left\langle A_{\mathrm{mss}}\right\rangle=120 \pm 1 \mu \mathrm{m}$ (inset of Fig. 3). Consequently, the micro-stick-slip period scales as $T_{\mathrm{mss}}=$ $\left\langle A_{\mathrm{mss}}\right\rangle / v_{m}$ (dashed line in Fig. 3) [18]. Thoroddsen et al. [16] found a larger constant value, probably due to the different adhesive-substrate joint studied. We also note that, for $v_{m}>5 \pm 1 \mathrm{~m} \mathrm{~s}^{-1}$, the micro-stick-slip period becomes smaller than the time for a transverse fracture to cross the tape width. Thus, for these $v_{m}$, several transverse fractures shifted in time of $T_{m s s}$ may be observed [16].

We performed additional experiments in order to image the peeled tape profile (Fig. 1) at 150000 frames/s and $512 \times 80 \mathrm{pixel}^{2}$, with a resolution of $5 \mu \mathrm{m} /$ pixel. Figure 4(a) shows the profiles extracted at intervals of $6.7 \mu$ s for an experiment at $V=0.73 \mathrm{~m} \mathrm{~s}^{-1}$ and $L=1 \mathrm{~m}$. After the macrostick phase during which the peeling front propagates slowly (about $0.04 \mathrm{~m} \mathrm{~s}^{-1}$, here), leading to a dense region of profiles on the right of Fig. 4(a), we observe the periodic micro-stick-slip dynamics during the macroslip phase: the tape curvature increases before decreasing suddenly in less than $6.7 \mu \mathrm{s}$, and so on. This drop of the ribbon curvature implies a release of its bending energy. In order to estimate the corresponding local energy release, we compute for each profile the angle $\alpha$ between the substrate plane and the tangent to the peeled tape as a function of the curvilinear abscissa $s$ from the detachment front [as defined

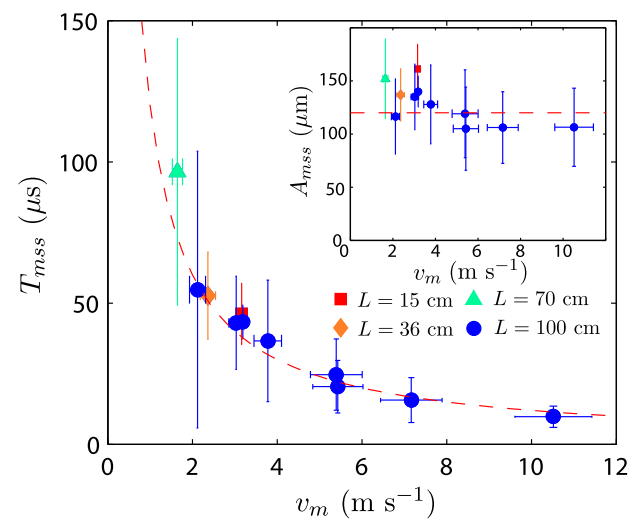

FIG. 3 (color online). Mean micro-stick-slip duration $T_{m s s}$ and amplitude $A_{m s s}$ (inset) as function of $v_{m}$ for various $L$. The dashed lines represent $T_{m s s}=\left\langle A_{m s s}\right\rangle / v_{m}$ and $A_{m s s}=\left\langle A_{m s s}\right\rangle$ (inset), with $\left\langle A_{m s s}\right\rangle=120 \mu \mathrm{m}$. in the inset of Fig. 4(b)]. Using the Elastica theory [24] (with a simple clamping boundary condition at the peeling front $s=0$ ) for a force $F$ pulling the tape extremity with an angle $\theta$ (see Supplemental Material [21]), one can show that $\alpha(s)=\theta-4 \arctan \left[e^{-s / r} \tan (\theta / 4)\right]$, where $r=\sqrt{E I / F}$, with $E$ the ribbon tensile modulus, and $I=e^{3} b / 12$, its second area moment. Fitting each experimental profile $\alpha(s)$, we extract the adjustable parameter, the peeling force $F$, as a function of time. A typical example of its evolution is shown in Fig. 4(b) for the experiment of Fig. 4(a). At the end of the macrostick, the force suddenly drops from about $3.5 \mathrm{~N}$ to approximately $0.5 \mathrm{~N}$ at the beginning of the macroslip phase. Then, the peeling force $F(t)$ displays quasiperiodic oscillations with slow increases followed by sudden drops of amplitude typically about $0.4 \mathrm{~N}$. These force oscillations are synchronized with the periodic propagation of the transverse fractures and therefore with the whole micro-stick-slip dynamics. Their amplitude is nearly independent of the front velocity $v_{m}$ (checked up to $4 \mathrm{~m} \mathrm{~s}^{-1}$ only, due to difficulties to image properly the tape profiles at larger $v_{m}$ ). For each micro-stick-slip cycle, we compute the variation of the bending energy $\Delta E_{c}$ associated to the drop of the peeling force using $E_{c}=$ $4 \sqrt{E I F} \sin ^{2} \theta / 4$ [25]. Assuming that this energy release allows the front to advance of a step $A_{\mathrm{mss}}$, we estimate an
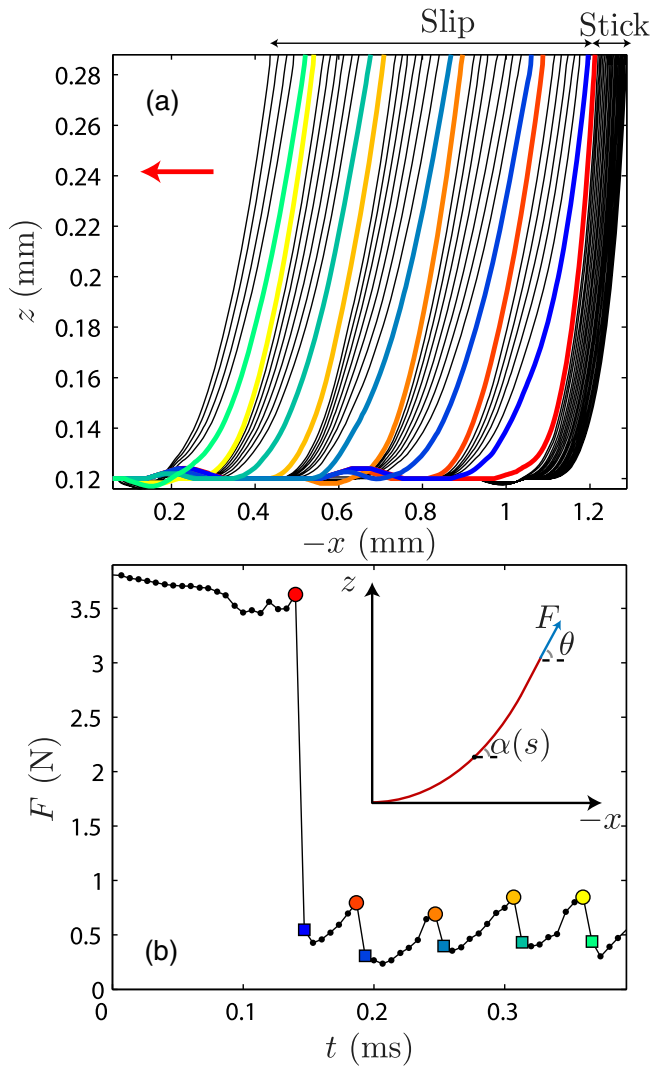

FIG. 4 (color online). Ribbon profile extracted at intervals of $6.7 \mu \mathrm{s}$ (a) and corresponding peeling force time evolution (b) when peeling at $V=0.73 \mathrm{~m} \mathrm{~s}^{-1}, L=1 \mathrm{~m}$. 
effective fracture energy associated to the micro-stick-slip regime as $\Gamma_{\mathrm{mss}}=\Delta E_{c} /\left(b A_{\mathrm{mss}}\right)$. We measure $\Gamma_{m s s}=$ $13.5 \pm 1.3 \mathrm{~J} / \mathrm{m}^{2}$, independently of $v_{m}$ (neither $A_{\mathrm{mss}}$ nor $\Delta E_{c}$ vary significantly with $v_{m}$ ), which is close to the steady state macroscopic fracture energy $G(V)$ at its local minimum observed for $V \simeq 19 \mathrm{~m} \mathrm{~s}^{-1}$ [11].

Interestingly, at the scale of the tape curvature close to the detachment front, $r=\sqrt{E I / F} \simeq 330 \mu \mathrm{m}$, just before the force drops, the ribbon of linear mass density $\mu=$ $(8.5 \pm 0.15) \times 10^{-4} \mathrm{~kg} \mathrm{~m}^{-1}$ has a natural bending vibration frequency of the order of $\sqrt{E I / \mu r^{4}}=93 \mathrm{kHz}$ [26]. This characteristic frequency sets a lower bound for the time scale for the release of the tape bending energy, in agreement with the highest micro-stick-slip frequency we measured. We also note that, following the force drop at the end of the macrostick, a decompression wave will propagate along the tape at $c=\sqrt{E e b / \mu} \simeq 1000 \mathrm{~m} \mathrm{~s}^{-1}$, converting elastic stretch energy of the tape into kinetic energy behind the wave front. This kinetic energy allows for a local reloading of the ribbon at an almost constant velocity after each microslip.

To conclude, we have shown that the small-scale highfrequency instability of adhesive tape peeling corresponds to a micro-stick-slip dynamics fundamentally different from the macroscopic stick-slip instability. The regular propagation of rapid transverse cracks which creates the micro-stickslip spatiotemporal pattern appears as a purely dynamic fracture instability. We have shown that the velocity $v_{m}$ of the peeled tape just beyond the bending region close to the peeling front is the control parameter of the micro-stick-slip instability, which is driven by the periodic release of the bending energy. The bending elasticity allows for the detachment front velocity to be instantly different from the tape velocity $v_{m}$ beyond the bending region (but equal on average). Further work is needed to understand the interplay between the release of the elastic stretch energy of the whole free standing tape, driving the macro-stick-slip, and the fast release of the ribbon bending energy close to the peeling front.

We thank D. Le Tourneau for his help in the design of the setup, O. Ramos for his help in the early stage of the experiments, and C. Creton and R. Villey for enlightening discussions. This work has been supported by the Research Federation "A. M. Ampère" (FRAMA) and the French ANR through Grant "STICKSLIP" No. 12BS09-014. *stephane.santucci@ens-lyon.fr

[1] T. Baumberger and C. Caroli, Adv. Phys. 55, 279 (2006).

[2] I. Svetlizky and J. Fineberg, Nature 509, 205 (2014).

[3] J. Fineberg and M. Marder, Phys. Rep. 313, 1 (1999).

[4] J. L. Gardon, J. Appl. Polym. Sci. 7, 625 (1963).

[5] D. W. Aubrey, G. N. Welding, and T. Wong, J. Appl. Polym. Sci. 13, 2193 (1969).

[6] J. L. Racich and J. A. Koutsky, J. Appl. Polym. Sci. 19, 1479 (1975).

[7] M. Barquins, B. Khandani, and D. Maugis, C.R. Acad. Sci. Ser. 2 303, 1517 (1986).

[8] D. Maugis and M. Barquins, Adhesion 12, edited by K. Allen (Elsevier ASP, London, 1988), pp. 205-222.

[9] G. Ryschenkow and H. Arribart, J. Adhes. 58, 143 (1996).

[10] M. Gandur, M. Kleinke, and F. Galembeck, J. Adhes. Sci. Technol. 11, 11 (1997).

[11] M. Barquins and M. Ciccotti, Int. J. Adhes. Adhes. 17, 65 (1997).

[12] M. Ciccotti, B. Giorgini, D. Vallet, and M. Barquins, Int. J. Adhes. Adhes. 24, 143 (2004).

[13] R. De and G. Ananthakrishna, Phys. Rev. E 70, 046223 (2004).

[14] P.-P. Cortet, M. Ciccotti, and L. Vanel, J. Stat. Mech. (2007) P03005.

[15] R. De, A. Maybhate, and G. Ananthakrishna, Eur. Phys. J. B 61, 475 (2008).

[16] S. T. Thoroddsen, H. D. Nguyen, K. Takehara, and T. G. Etoh, Phys. Rev. E 82, 046107 (2010).

[17] P.-P. Cortet, M.-J. Dalbe, C. Guerra, C. Cohen, M. Ciccotti, S. Santucci, and L. Vanel, Phys. Rev. E 87, 022601 (2013).

[18] J. O. Marston, P. W. Riker, and S. T. Thoroddsen, Sci. Rep. 4, 4326 (2014).

[19] M.-J. Dalbe, S. Santucci, P.-P. Cortet, and L. Vanel, Soft Matter 10, 132 (2014).

[20] M.-J. Dalbe, S. Santucci, L. Vanel, and P.-P. Cortet, Soft Matter 10, 9637 (2014).

[21] See Supplemental Material at http://link.aps.org/ supplemental/10.1103/PhysRevLett.115.128301 for a series of videos of typical peeling experiments.

[22] The stick-slip onset velocity $V_{a}$ is slightly larger than in previous experiments $[11,19]$ where the same adhesive was peeled directly from its roll. This is due to the cleaning procedure of the tape backing used as a substrate, which removes its release coating and thus increases the adhesion.

[23] Y. Yamazaki and A. Toda, Physica D 214, 120 (2006).

[24] A. E. H. Love, A Treatise on the Mathematical Theory of Elasticity (Dover Publications, New York, 1944).

[25] B. Roman, Int. J. Fract. 182, 209 (2013).

[26] W. Weaver, Jr., S. P. Timoshenko, and D. H. Young, Vibration Problems in Engineering (Wiley, New York, 1990). 